\title{
Association of Dietary Variety Status and Sarcopenia in Korean Elderly
}

\author{
Hee-Sook Lim \\ Department of Food and Nutrition, Yeonsung University, Anyang, Korea
}

\author{
Corresponding author \\ Hee-Sook Lim \\ Department of Food and Nutrition, Yeonsung \\ University, 34 Yanghwa-ro, Manan-gu, \\ Anyang 14011, Korea \\ Tel: +82-31-441-1468 \\ Fax: +82-31-441-1349 \\ E-mail: limhs@yeonsung.ac.kr
}

Received: January 28, 2020

Revised: May 15, 2020

Accepted: May 16, 2020
Background: Sarcopenia is associated with a variety of factors including age, diseases, exercise, and heredity. In particular, diet is known to affect changes in muscle mass loss. Methods: The purpose of this study was to analyze the diversity of food intake according to the presence of sarcopenia in elderly people over 65 years old using the 2008 to 2011 Korea National Health and Nutrition Examination Survey. The differences in subjects' general characteristics, lifestyle, and intake of 15 food groups were compared, and the risk odds ratio (OR) for sarcopenia was calculated for each food intake by gender. Results: The proportion of sarcopenia was $27.7 \%$ for males and $24.3 \%$ for females. The sarcopenia group had significantly lower intakes of nuts and seeds, meats, and milks than the non-sarcopenia group in males. The females had significantly lower intake of fruits, milks, and beverages in non-sarcopenia group. The dietary diversity score was significantly lower in females with sarcopenia than non-sarcopenia group. Regression analysis of the risk OR for sarcopenia according to food group intake showed that the subjects in the lowest tertile ( $<31.1 \mathrm{~g}$ ) had a 1.83 times (95\% confidence interval [Cl], 1.13-2.42) risk for sarcopenia in males. In case of milks intake of females, the risk for sarcopenia increased 1.39 times $(95 \% \mathrm{Cl}, 1.11-1.86)$ in subjects with the lowest tertile $(<30.1 \mathrm{~g})$. Conclusions: Dietary diversity status was the most vulnerable to female with sarcopenia. Sarcopenia was associated with meat intake in male and milk intake in female.

Key Words: Aged · Diet, healthy $\cdot$ Food quality $\cdot$ Sarcopenia

\section{INTRODUCTION}

It is estimated that the population aging in Korea is getting more progressed from $10.2 \%$ in 2005 to $35.6 \%$ in 2045 , and the progress rate of aging is the fastest among Organisation for Economic Co-operation and Development countries.[1] Therefore, healthcare for the elderly aged 65 or older is very important at the national level beyond the individual perspectives. In the process of aging, muscle loss is known to progress gradually from their 40 s, and the elderly suffer from changes in body composition such as a decrease in muscle mass and an increase in body fat mass as well as deterioration of the muscle quality.[2,3] This not only interferes with protein metabolism in the muscles but also cause insulin resistance.[4]

Nutrition issues are somewhat different according to age group. While overnutrition or nutritional imbalance are the issues among young adults, the elderly people still have a deficiency of food and nutrient intake.[5] Sarcopenia refers to a

\section{Mineral Research}

This is an Open Access article distributed under the terms of the Creative Commons Attribution Non-Commercial License (https://creativecommons.org/licenses/by-nc/4.0/ which permits unrestricted non-commercial use, distribution, and reproduction in any medium, provided the origina work is properly cited.

\section{KSBMR}


decrease in muscle mass and muscle strength, which is common in weak elderly people who have less physical activity and exercise, and poor nutrition.[6] Malnutrition caused by decrease in nutrient intake promotes the progression of sarcopenia.[7] Previous studies on the association between sarcopenia and nutrients showed that inadequate protein intake negatively affected the synthesis of muscle proteins in the elderly.[8,9] It is reported that the highest protein intake group had about $40 \%$ less muscle loss than the low protein intake group.[8] Muscle loss is accompanied by a decrease in the vitamin $D$ receptor in the muscle, so increasing the level of vitamin $D$ in the blood is an important factor in maintaining high muscle mass.[10] In addition, the concentration of antioxidants in the blood is positively correlated with physical activity and muscle strength, and the effects of leucine, which positively affects muscle protein synthesis, can be inhibited by oxidative stress, which can be improved by antioxidant nutrients.[11,12] As a result, when there are various kinds of foods that contain enough nutrients, it is possible to consume balanced nutrients. Many studies have been conducted on the nutritional intake of the elderly in Korea, but few studies have been conducted on sarcopenia.

The purpose of this study was to examine the sarcopenia status of the elderly and to analyze the qualitative aspects of diet through the evaluation of food diversity according to the sarcopenic status and to provide the basic data necessary for setting guidelines for the reduction of muscle.

\section{METHODS}

\section{Study subject}

This study used the data of 2008 to 2011 Korea National Health and Nutrition Examination Survey (KNHANES), which contain the body composition data which are required to calculate muscle mass. The subjects of the study were the elderly over 65 . A total of 3,350 people were extracted, by excluding those under 65 years of age, those who had missing analytical variables, or those whose daily calorie intake is less than $500 \mathrm{kcal}$ or over $5,000 \mathrm{kcal}$. Out of the subjects, males were $42.6 \%$, and females were $57.4 \%$. A total of 862 suspected sarcopenia were classified. The method proposed by Janssen et al. [13] was used as a standard of diagnosis of sarcopenia. When the muscle mass excluding bones and fats of limbs measured by dual energy $X$-ray absorptiometry
(DISCOVERY-W; Hologic Inc., Bedford, MA, USA) divided by weight in the form of percent is under the twice of standard deviation (SD), it was classified as sarcopenia.[14]

\section{Study variables}

In body mass index (BMI), household income (low, middle-low, middle-high, high), marital status (married, single), education level (elementary school or below, middle school, high school, university or above), smoking status (current smoker, past smoker, no smoker), alcohol consumption (yes or no), activity limitation (yes or no) and subjective health status (good, so-so, bad) were used as demographic factors and lifestyle data in the KNHANES. Marriage variable in the marital status includes current marriage, separation, divorce, and bereavement.

Food intake analysis is based on the guideline of 15 food groups (cereals, potato and starches, sugars and sweeteners, pulses, nuts and seeds, vegetables, fungi and mushrooms, fruits, meats, eggs, fish and shellfishes, seaweeds, milks, oil and fat, and beverages).[15] The number of foods consumed during the day was calculated.[15] This is called the dietary variety score (DVS) and is a tool for evaluating the variety of diet.[16] Dietary diversity score (DDS) was calculated by classifying the foods consumed by the subjects into 5 major food groups (cereals, fish and meats, vegetables, fruits, and dairy groups) and assigning one point for each food group consumed above the minimum during the day.[15,16]

\section{Statistical analysis}

Complex sample analysis was performed to the national survey data using the weights, stratified variables, and cluster variables following the guidelines from the KNHANES to achieve results without biases. In order to reflect the sample design information of non-respondents of specific survey variables without missing, the standard error of the estimate was not underestimated by processing the missing value as a valid value in the analysis of the complex sample. The mean and SD were calculated using SPSS software program version 18.0 (SPSS Inc., Chicago, IL, USA). All data were reported as mean \pm SD or as percentages. Groups comparisons used $\chi^{2}$ tests for qualitative variables and independent $t$-test for quantitative variables. Multiple logistic regression analyses were used to measure the association between the tertiles of food intake amount and the 
presence of sarcopenia and was performed to compute the odds ratios (ORs) and 95\% confidence intervals (Cis). All $P$-values of less than 0.05 were considered to indicate statistical significance.

\section{RESULTS}

1. Demographic factors and lifestyle of the subjects

The proportion of sarcopenia was $\mathbf{2 7 . 7 \%}$ for males and $24.3 \%$ for females. However, although not mentioned in the Table 1. In the BMI, the sarcopenia group was significantly lower than the non-sarcopenia group. There were no significant variables among males, but females showed a significant difference according to the marital status, education level, and energy intake (Table 1).

\section{Dietary variety status and food intakes from each food group of the subject}

Among the 15 food groups, the sarcopenia group had significantly lower intakes of nuts and seeds, meats, and milks than the non-sarcopenia group in males. The females

Table 1. General characteristics and lifestyle factors by sarcopenia status

\begin{tabular}{|c|c|c|c|c|c|c|}
\hline \multirow[b]{2}{*}{ Variable } & \multicolumn{3}{|c|}{ Male } & \multicolumn{3}{|c|}{ Female } \\
\hline & $\begin{array}{c}\text { Non-sarcopenia } \\
(n=1,032)\end{array}$ & $\begin{array}{c}\text { Sarcopenia } \\
(n=395)\end{array}$ & $P$-value ${ }^{\mathrm{a})}$ & $\begin{array}{c}\text { Non-sarcopenia } \\
(n=1,456)\end{array}$ & $\begin{array}{c}\text { Sarcopenia } \\
(n=467)\end{array}$ & $P$-value ${ }^{\text {a) }}$ \\
\hline Age (yr) & $69.4 \pm 8.6$ & $68.3 \pm 6.9$ & 0.426 & $66.2 \pm 9.1$ & $72.4 \pm 10.3$ & 0.004 \\
\hline BMI $\left(\mathrm{kg} / \mathrm{m}^{2}\right)$ & $25.7 \pm 3.4$ & $23.4 \pm 2.1$ & $<0.001$ & $24.0 \pm 3.1$ & $21.6 \pm 2.8$ & $<0.001$ \\
\hline Income & & & 0.310 & & & 0.194 \\
\hline Low & 26.5 & 24.5 & & 25.1 & 23.0 & \\
\hline Middle-low & 25.8 & 26.2 & & 25.8 & 24.5 & \\
\hline Middle-high & 24.6 & 23.9 & & 25.3 & 27.1 & \\
\hline High & 23.1 & 25.4 & & 23.8 & 25.4 & \\
\hline Marital status & & & 0.356 & & & 0.027 \\
\hline Married & 91.3 & 89.6 & & 93.9 & 87.2 & \\
\hline Single & 8.7 & 10.4 & & 6.1 & 12.8 & \\
\hline Education level & & & 0.489 & & & 0.004 \\
\hline$\leq$ Elementary & 62.4 & 60.3 & & 70.7 & 73.3 & \\
\hline Middle & 16.2 & 17.5 & & 12.0 & 13.8 & \\
\hline High & 17.0 & 16.4 & & 13.1 & 11.6 & \\
\hline$\geq$ College & 4.4 & 5.8 & & 4.2 & 1.3 & 0.004 \\
\hline Smoking status & & & 0.251 & & & 0.572 \\
\hline Current smoker & 23.3 & 25.6 & & 8.9 & 9.6 & \\
\hline Past smoker & 15.9 & 17.7 & & 2.0 & 1.4 & \\
\hline No smoker & 60.8 & 56.7 & & 89.1 & 89.0 & \\
\hline Alcohol consumption & & & 0.663 & & & 0.419 \\
\hline Yes & 55.9 & 54.4 & & 15.5 & 14.2 & \\
\hline No & 44.1 & 45.6 & 0.663 & 84.5 & 85.8 & \\
\hline Activity limitation & & & 0.701 & & & 0.544 \\
\hline Yes & 26.4 & 27.1 & & 37.1 & 36.7 & \\
\hline No & 73.6 & 72.9 & & 62.9 & 63.3 & \\
\hline Subjective health status & & & 0.463 & & & 0.510 \\
\hline Good & 25.3 & 27.1 & & 25.8 & 24.7 & \\
\hline So-so & 44.6 & 43.2 & & 39.6 & 40.1 & \\
\hline Bad & 30.1 & 29.7 & & 34.6 & 35.2 & \\
\hline Energy intake (kcal) & $1,864.2 \pm 113.6$ & $1,756.4 \pm 91.8$ & 0.382 & $1,651.9 \pm 108.1$ & $1,438.0 \pm 56.3$ & 0.025 \\
\hline
\end{tabular}

The data is presented as mean \pm standard deviation or $\%$.

${ }^{\text {a) }} P$-value by $\chi^{2}$ test (categorical variables) and independent $t$-test (continuous variables).

$\mathrm{BMI}$, body mass index. 
Table 2. Dietary variety status and food intakes per day from each food group by sarcopenia status

\begin{tabular}{|c|c|c|c|c|c|c|}
\hline \multirow[b]{2}{*}{ Variable } & \multicolumn{3}{|c|}{ Male } & \multicolumn{3}{|c|}{ Female } \\
\hline & $\begin{array}{l}\text { Non-sarcopenia } \\
\quad(n=1,032)\end{array}$ & $\begin{array}{l}\text { Sarcopenia } \\
(n=395)\end{array}$ & $P$-value & $\begin{array}{l}\text { Non-sarcopenia } \\
(n=1,456)\end{array}$ & $\begin{array}{c}\text { Sarcopenia } \\
(n=467)\end{array}$ & $P$-value al \\
\hline \multicolumn{7}{|l|}{ Food group (g) } \\
\hline Cereals & $341.2 \pm 15.5$ & $331.8 \pm 20.8$ & 0.401 & $285.6 \pm 11.5$ & $283.9 \pm 14.8$ & 0.751 \\
\hline Potato and starches & $31.2 \pm 2.8$ & $30.4 \pm 6.7$ & 0.805 & $46.0 \pm 3.8$ & $39.5 \pm 8.1$ & 0.862 \\
\hline Sugars and sweeteners & $8.3 \pm 2.5$ & $7.5 \pm 1.7$ & 0.251 & $7.3 \pm 1.4$ & $7.5 \pm 1.6$ & 0.697 \\
\hline Pulses & $45.8 \pm 8.2$ & $43.4 \pm 11.1$ & 0.776 & $31.6 \pm 6.6$ & $29.3 \pm 5.3$ & 0.647 \\
\hline Nuts and seeds & $5.2 \pm 0.8$ & $3.1 \pm 0.4$ & 0.002 & $4.0 \pm 0.5$ & $3.5 \pm 0.9$ & 0.258 \\
\hline Vegetables & $398.8 \pm 10.7$ & $404.7 \pm 12.2$ & 0.456 & $330.4 \pm 8.9$ & $278.2 \pm 11.3$ & 0.154 \\
\hline Fungi and mushrooms & $5.0 \pm 0.7$ & $5.7 \pm 2.5$ & 0.158 & $3.8 \pm 0.5$ & $3.7 \pm 1.0$ & 0.597 \\
\hline Fruits & $120.7 \pm 11.5$ & $123.4 \pm 13.4$ & 0.208 & $190.6 \pm 13.5$ & $114.1 \pm 19.6$ & $<0.001$ \\
\hline Meats & $72.4 \pm 9.2$ & $51.8 \pm 12.7$ & 0.005 & $60.8 \pm 7.1$ & $53.1 \pm 10.2$ & 0.185 \\
\hline Eggs & $20.8 \pm 3.5$ & $23.1 \pm 5.4$ & 0.530 & $26.3 \pm 1.1$ & $11.4 \pm 2.6$ & 0.047 \\
\hline Fish and shellfishes & $77.3 \pm 6.2$ & $72.8 \pm 10.1$ & 0.295 & $46.3 \pm 5.3$ & $47.5 \pm 6.8$ & 0.749 \\
\hline Seaweeds & $6.7 \pm 1.1$ & $6.8 \pm 2.0$ & 0.833 & $6.8 \pm 1.0$ & $6.2 \pm 1.7$ & 0.660 \\
\hline Milks & $60.4 \pm 6.1$ & $38.0 \pm 11.5$ & $<0.001$ & $70.3 \pm 5.3$ & $32.3 \pm 9.4$ & $<0.001$ \\
\hline Oil and fat & $9.1 \pm 1.5$ & $8.4 \pm 1.7$ & 0.215 & $5.9 \pm 0.8$ & $6.1 \pm 0.5$ & 0.482 \\
\hline Beverages & $291.0 \pm 15.4$ & $286.3 \pm 22.5$ & 0.249 & $128.6 \pm 11.5$ & $59.6 \pm 16.2$ & $<0.001$ \\
\hline Dietary variety score & $9.51 \pm 0.2$ & $9.60 \pm 0.4$ & 0.751 & $9.70 \pm 0.7$ & $9.14 \pm 0.5$ & 0.082 \\
\hline Dietary diversity score & $3.50 \pm 0.0$ & $3.54 \pm 0.1$ & 0.848 & $3.62 \pm 0.1$ & $3.28 \pm 0.0$ & 0.007 \\
\hline
\end{tabular}

The data is presented as mean \pm standard deviation.

a) $P$-value by independent $t$-test (continuous variables).

had significantly lower intake of fruits, milks, and beverages in non-sarcopenia group. The DVS and DDS were not significantly different between non-sarcopenia and sarcopenia in males, but DDS was significantly lower in sarcopenia than non-sarcopenia group (Table 2).

\section{Multivariate analysis for associations \\ between sarcopenia and food intake status}

Logistic regression analyses were designed to figure out the prevalence of sarcopenia according to the tertiles of food group intakes. In case of meats intake of males, subjects in the lowest tertile $(<31.1 \mathrm{~g})$ had a 2.11 times $(95 \%$ $\mathrm{Cl}, 1.44-2.68)$ risk for sarcopenia, compared with those in the highest tertile $(\geq 55.3 \mathrm{~g}$ ) in the crude model. After further multiple adjustment for age, BMI, marital status, educational level, and energy intake factors, the ORs were remained statistically significant (tertile 3: OR, $1.83,95 \% \mathrm{Cl}$, 1.13-2.42 compared with tertile 1). In case of milks intake of females, the risk for sarcopenia increased 1.74 times (95\% Cl, 1.64-2.03) in subjects with the lowest tertile of milks intake $(<30.1 \mathrm{~g})$ compared with the highest tertile ( $\geq 51.9 \mathrm{~g}$ ). After adjustment for the same factors in model- ling males previously, the ORs were attenuated but remained significant (tertile 3: OR, 1.39, 95\% Cl, 1.11-1.86 compared with tertile 1) (Table 3).

\section{DISCUSSION}

We was identified food factors affecting sarcopenia in this study. The skeletal muscle mass is the main organ of the body's energy and oxygen consumption and keeps physical movement and posture smooth. It also serves as the main reservoir of nutrients such as amino acids and glucose. Therefore, retention of the skeletal muscle mass is in a close connection with constancy of energy metabolism and health maintenance.[17,18] Furthermore, studies focused on high loss subjects such as diabetes, cancer, elderly patients report that suitable maintenance of skeletal muscle mass improves response of medical treatment, quality of life and reduces mortality rate.[19,20] The skeletal muscle mass between men and women is somewhat different in each studies. In one longitudinal research that tracked Japanese elderly people for 12 years, the women's loss of skeletal muscle mass was higher than men's.[21] Ac- 
Table 3. Multiple linear regression models for the sarcopenia and food group intakes

\begin{tabular}{|c|c|c|c|c|}
\hline & \multicolumn{2}{|c|}{ Male } & \multicolumn{2}{|c|}{ Female } \\
\hline & Model $1^{\text {a) }}$ & Model $2^{\text {b) }}$ & Model $1^{\text {a) }}$ & Model $2^{\text {b) }}$ \\
\hline \multicolumn{5}{|c|}{ Nuts and seeds $(\mathrm{g} / \text { day })^{c)}$} \\
\hline Tertile 1 & 1.00 (reference) & 1.00 (reference) & 1.00 (reference) & 1.00 (reference) \\
\hline Tertile 2 & 1.01 (0.8.-1.29) & $1.03(0.71-1.31)$ & $1.02(0.78-1.50)$ & $0.99(0.77-1.26)$ \\
\hline Tertile 3 & $1.12(0.87-1.43)$ & $1.09(0.84-1.40)$ & $1.03(0.81-1.25)$ & $1.01(0.79-1.31)$ \\
\hline \multicolumn{5}{|c|}{ Friuts $(g / \text { day })^{d)}$} \\
\hline Tertile 1 & 1.00 (reference) & 1.00 (reference) & 1.00 (reference) & 1.00 (reference) \\
\hline Tertile 2 & $0.98(0.78-1.24)$ & $0.99(0.81-1.25)$ & $1.11(0.85-1.24)$ & $1.06(0.51-1.28)$ \\
\hline Tertile 3 & $1.07(0.86-1.33)$ & $1.04(0.82-1.31)$ & $1.14(0.95-1.26)^{\mathrm{h})}$ & $1.10(0.92-1.88)$ \\
\hline \multicolumn{5}{|c|}{ Meats $(g / \text { day })^{e)}$} \\
\hline Tertile 1 & 1.00 (reference) & 1.00 (reference) & 1.00 (reference) & 1.00 (reference) \\
\hline Tertile 2 & $1.40(0.98-1.85)^{\mathrm{i}}$ & $1.49(0.99-1.71)^{i)}$ & $1.03(0.92-1.07)$ & $1.03(0.85-1.20)$ \\
\hline Tertile 3 & $2.11(1.44-2.68)^{i)}$ & $1.83(1.13-2.42)^{i)}$ & $1.06(0.94-1.15)$ & $1.04(0.88-1.16)$ \\
\hline \multicolumn{5}{|c|}{ Milks (g/day) $)^{\dagger}$} \\
\hline Tertile 1 & 1.00 (reference) & 1.00 (reference) & 1.00 (reference) & 1.00 (reference) \\
\hline Tertile 2 & $1.03(0.55-1.29)$ & $1.01(0.80-1.22)$ & $1.20(1.11-1.27)^{i)}$ & $1.16(1.11-1.22)^{i)}$ \\
\hline Tertile 3 & $1.08(0.86-1.49)$ & $1.09(0.87-1.44)$ & $1.74(1.64-2.03)^{\mathrm{i})}$ & $1.39(1.11-1.86)^{i)}$ \\
\hline \multicolumn{5}{|c|}{ Beverages $(\mathrm{g} / \text { day })^{g)}$} \\
\hline Tertile 1 & 1.00 (reference) & 1.00 (reference) & 1.00 (reference) & 1.00 (reference) \\
\hline Tertile 2 & $1.05(0.74-1.33)$ & $0.97(0.77-1.14)$ & $0.96(0.85-1.05)$ & $0.99(0.95-1.03)$ \\
\hline Tertile 3 & $1.01(0.83-1.19)$ & $1.00(0.90-1.11)$ & $1.03(0.98-1.07)$ & $1.04(0.97-1.11)$ \\
\hline
\end{tabular}

The data is presented as odds ratios ( $95 \%$ confidence interval).

${ }^{\text {al } M o d e l ~ 1: ~ c r u d e ~ m o d e l . ~}{ }^{b}$ Model 2: adjusted for age, BMI, marrital status, educational level, and energy intake. ${ }^{\mathrm{cl}}$ Tertile 1: male ( $\left.\geq 4.1\right)$, female ( $\left.\geq 3.6\right)$. Tertile 2: male (1.0-4.0), female (2.2-3.5). Tertile 3: male $(<1.0)$, female $(<2.2)$. ${ }^{d}$ Tertile 1: male $(\geq 86.3)$, female $(\geq 120.6)$. Tertile 2: male (25.4-86.2),

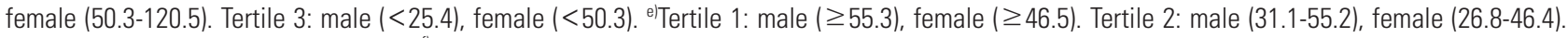
Tertile 3: male $(<31.1)$, female $(<26.8)$. ${ }^{\text {fl}}$ Tertile 1: male $(\geq 49.7)$, female $(\geq 51.9)$, Tertile 2: male (28.5-49.6), female (30.1-51.8). Tertile 3: male $(<28.5)$,

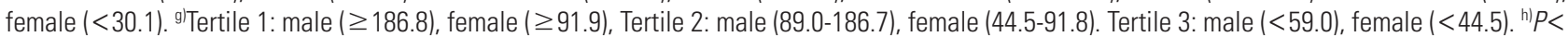
0.05. ${ }^{\text {i) }} P<0.01$. ${ }^{\text {j) }} P<0.001$.

cording to Davidson et al. [22] the decrease rate was nearly twice higher in men than women for healthy adults. In this study, male's skeletal muscle mass loss was slightly higher.

In the results about diversity of food intake, male's intake of nuts and seeds, milks, and meats was slightly lower in sarcopenia group. In females, fruits, milks, and beverages intake was low, and DDS was also slightly low. Low food variety of elderly people was reported: $35.2 \%, 59.3 \%$, and $22.4 \%$ of subjects consumed no dairy products, fruits, and vegetables, respectively.[23] A cross-sectional study found that in women, a higher fruit and vegetable variety score was associated with higher mid-arm muscle area.[24] Therefore, a screening test that assess food variety in elderly people was recommended to be incorporated at a primary care level to identify older adults most at risk of a poor quality diet.[23] In Foote et al. [25] the quality and appropriateness of nutrition intake gets lower as the older the elderly is. It also stressed the importance of calcium and vitamin D intake considering osteoporosis and bone fractures commonly happening in older people.[25] The Kumagai and colleague [26] proposed that the total food score of the elderly would have lower risk of reduction in high strength body function, and the reduction of diversity of food intake would make high risk of sarcopenia. Muscle mass was also said to be related to dietary pattern. Mediterranean diet which consume vitamin C, selenium, and magnesium could influence muscle health, and it was reported that this can be different by race and nations' food culture.[27] For muscle mass increasing, enough nutrition support from diet is most important, however it is also necessary to intake rich protein that has full essential amino acid and calcium which is needed for synthesizing myoprotein.[28] This study also shows that major foods which contains nutrients that affect muscle mass showed deficient result in 
sarcopenia group. In addition, in multiple regression analysis that adjusted many factors showed proper intake of meat in males and sufficient intake of milk in females significantly reduces the risk ratio of sarcopenia. Therefore, it seems to be essential to improve quality of food through diverse and sufficient food intake to prevent sarcopenia. In the elderly, it is necessary to distinguish male and female food that each needs special attention.

This study has several limitations. As it is domestic crosssectional study, it can't explain cause-effect relationship of sarcopenia and the result of food intake state. It is difficult to reflect individual's usual dietary intake state since dietary assessment method was measured by 24-hr dietary intake data. In addition, for accurate diagnose of sarcopenia, the classification of subjects should be carried out through new classification using muscle function and a complementary research is required since accuracy may be reduce in limited number of samples.

\section{CONCLUSIONS}

To sum up this research, there was a difference in male and female's intake of food groups depending whether they had sarcopenia in Korean elderly. Both male and female who have sarcopenia had lack intake in milks, and DDS of female with sarcopenia was lowest. It was confirmed that meat intake in male and milk intake in female had an significant correlation on sarcopenia development. For the elderly, nutritional education and management are necessary to maintain a balanced nutrition status through the proper food choice and intake. We expect that it can be used as a basis for preparing dietary guidelines to prevent sarcopenia in elderly people.

\section{DECLARATIONS}

\section{Ethics approval and consent to participate}

This study complies with the Declaration of Helsinki and was performed according to ethics committee approval.

\section{Conflict of interest}

No potential conflict of interest relevant to this article was reported.
ORCID

Hee-Sook Lim https://orcid.org/0000-0003-0745-8906

\section{REFERENCES}

1. Park MH. Comparative study on ageing society: Korea and EU. Korean J EU Stud 2011;16:99-126.

2. Hughes VA, Frontera WR, Roubenoff $R$, et al. Longitudinal changes in body composition in older men and women: role of body weight change and physical activity. Am J Clin Nutr 2002;76:473-81.

3. Poggiogalle E, Lubrano C, Sergi G, et al. Sarcopenic obesity and metabolic syndrome in adult Caucasian subjects. J Nutr Health Aging 2016;20:958-63.

4. Lee CG, Boyko EJ, Strotmeyer ES, et al. Association between insulin resistance and lean mass loss and fat mass gain in older men without diabetes mellitus. J Am Geriatr Soc 2011;59:1217-24.

5. Tian HY, Qiu R, Jing LP, et al. Alternate Mediterranean diet score is positively associated with skeletal muscle mass index in middle-aged adults. Br J Nutr 2017;117:1181-8.

6. Yoon JR, Ha GC, Kang SJ, et al. Effects of 12-week resistance exercise and interval training on the skeletal muscle area, physical fitness, and mental health in old women. J Exerc Rehabil 2019;15:839-47.

7. Gautsch TA, Kandl SM, Donovan SM, et al. Growth hormone promotes somatic and skeletal muscle growth recovery in rats following chronic protein-energy malnutrition. J Nutr 1999;129:828-37.

8. Liao CD, Chen HC, Huang SW, et al. The role of muscle mass gain following protein supplementation plus exercise therapy in older adults with sarcopenia and frailty risks: a systematic review and meta-regression analysis of randomized trials. Nutrients 2019;11:1713.

9. Gade J, Beck AM, Bitz C, et al. Protein-enriched, milk-based supplement to counteract sarcopenia in acutely ill geriatric patients offered resistance exercise training during and after hospitalisation: study protocol for a randomised, double-blind, multicentre trial. BMJ Open 2018;8:e019210.

10. Remelli F, Vitali A, Zurlo A, et al. Vitamin D deficiency and sarcopenia in older persons. Nutrients 2019;11:2861.

11. Damiano S, Muscariello E, La Rosa G, et al. Dual role of reactive oxygen species in muscle function: can antioxidant dietary supplements counteract age-related sarcopenia? Int J Mol Sci 2019;20:3815. 
12. Chaput JP, Lord C, Cloutier M, et al. Relationship between antioxidant intakes and class I sarcopenia in elderly men and women. J Nutr Health Aging 2007;11:363-9.

13. Janssen I, Heymsfield SB, Ross R. Low relative skeletal muscle mass (sarcopenia) in older persons is associated with functional impairment and physical disability. J Am Geriatr Soc 2002;50:889-96.

14. Kim KM, Jang HC, Lim S. Differences among skeletal muscle mass indices derived from height-, weight-, and body mass index-adjusted models in assessing sarcopenia. Korean J Intern Med 2016;31:643-50.

15. Randall E, Nichaman MZ, Contant CF, Jr. Diet diversity and nutrient intake. J Am Diet Assoc 1985;85:830-6.

16. Kant AK, Block G, Schatzkin A, et al. Dietary diversity in the US population, NHANES II, 1976-1980. J Am Diet Assoc 1991;91:1526-31.

17. Wilkinson DJ, Piasecki M, Atherton PJ. The age-related loss of skeletal muscle mass and function: measurement and physiology of muscle fibre atrophy and muscle fibre loss in humans. Ageing Res Rev 2018;47:123-32.

18. Heymsfield SB, Gonzalez MC, Lu J, et al. Skeletal muscle mass and quality: evolution of modern measurement concepts in the context of sarcopenia. Proc Nutr Soc 2015;74: 355-66.

19. Lidoriki I, Schizas D, Mpaili E, et al. Associations between skeletal muscle mass index, nutritional and functional status of patients with oesophago-gastric cancer. Clin Nutr ESPEN 2019;34:61-7.

20. Doherty TJ. The influence of aging and sex on skeletal mus- cle mass and strength. Curr Opin Clin Nutr Metab Care 2001;4:503-8.

21. Shimokata $H$, Ando F, Yuki A, et al. Age-related changes in skeletal muscle mass among community-dwelling Japanese: a 12-year longitudinal study. Geriatr Gerontol Int 2014;14 Suppl 1:85-92.

22. Davidson LE, Kelley DE, Heshka S, et al. Skeletal muscle and organ masses differ in overweight adults with type 2 diabetes. J Appl Physiol (1985) 2014;117:377-82.

23. Lo YT, Chang YH, Lee MS, et al. Dietary diversity and food expenditure as indicators of food security in older Taiwanese. Appetite 2012;58:180-7.

24. Bloom I, Shand C, Cooper C, et al. Diet quality and sarcopenia in older adults: a systematic review. Nutrients 2018; 10:308.

25. Foote JA, Murphy SP, Wilkens LR, et al. Dietary variety increases the probability of nutrient adequacy among adults. J Nutr 2004;134:1779-85.

26. Kumagai $\mathrm{S}$, Watanabe $\mathrm{S}$, Shibata $\mathrm{H}$, et al. Effects of dietary variety on declines in high-level functional capacity in elderly people living in a community. Nihon Koshu Eisei Zasshi 2003;50:1117-24.

27. Silva TRD, Martins CC, Ferreira LL, et al. Mediterranean diet is associated with bone mineral density and muscle mass in postmenopausal women. Climacteric 2019;22:162-8.

28. Bonjour JP, Kraenzlin M, Levasseur R, et al. Dairy in adulthood: from foods to nutrient interactions on bone and skeletal muscle health. J Am Coll Nutr 2013;32:251-63. 
